\title{
PROBING BEYOND THE EPOCH OF HYDROGEN REIONIZATION WITH 21 CENTIMETER RADIATION
}

\author{
Benedetta Ciardi $^{1}$ \& Piero Madau $^{2,3}$ \\ submitted to the ApJ
}

\begin{abstract}
We use numerical simulations of hydrogen reionization by stellar sources in the context of $\Lambda$ CDM cosmogonies to investigate the $21(1+z) \mathrm{cm}$ radio signal expected from the diffuse intergalactic medium (IGM) prior to the epoch of reionization breakthrough at redshift $z_{\text {ion. }}$. Two reionization scenarios are analyzed in details: an 'early reionization' case with $z_{\text {ion }} \approx 13$, consistent with the recent discovery by the WMAP satellite of a large optical depth to Thomson scattering, and a 'late reionization' case with $z_{\text {ion }} \approx 8$. It is a generic prediction of these models that the background of Ly $\alpha$ photons produced by the early generation of stars which ultimately ionize the universe will be sufficiently intense to make intergalactic neutral hydrogen visible against the cosmic microwave background during the 'grey age', i.e. $z_{\text {ion }} \lesssim z \lesssim 20$. Depending on the redshift of reionization breakthrough, broad-beam observations at frequencies $\lesssim 150 \mathrm{MHz}$ (below $100 \mathrm{MHz}$ for the 'early reionization' scenario) with the next generation of radio telescopes should reveal angular fluctuations in the sky brightness temperature in the range 5-20 $\mathrm{mK}(1 \sigma)$ on scales below 5 arcmin.
\end{abstract}

Subject headings: cosmology: theory - diffuse radiation - galaxies: evolution - intergalactic medium

\section{INTRODUCTION}

Despite much recent theoretical and observational progress in our understanding of the formation of early cosmic structures and the high redshift universe, many fundamental questions remain, at best, only partially answered. We know that at least some galaxies and quasars were already shining when the universe was less than $10^{9}$ yr old. But when did the first luminous objects form, and what was their impact on the surrounding intergalactic gas? While the excess $\mathrm{H}$ I absorption measured in the spectra of $z \sim 6$ quasars in the Sloan Digital Sky Survey has been interpreted as the signature of the trailing edge of the cosmic reionization epoch (Becker et al. 2001; Fan et al. 2002), the recent analysis of the first year data from the Wilkinson Microwave Anisotropy Probe (WMAP) satellite infers a mean optical depth to Thomson scattering $\tau_{e} \sim 0.17$, suggesting that the universe was reionized at higher redshift (Kogut et al. 2003; Spergel et al. 2003). This is an indication of significant star-formation activity at very early times. In currently popular cosmological scenarios it is an early generation of subgalactic stellar systems - aided perhaps by a seed population of accreting black holes in their nuclei - that may have generated the ultraviolet radiation that ended the cosmic 'dark ages' and reionized most of the hydrogen in the universe around redshift $8-15$. The detailed thermal history of the baryons during and soon after these crucial formative stages depends on the power-spectrum of density fluctuations on small scales and on a complex network of poorly understood 'feedback' mechanisms, and remains one of the missing link in galaxy formation and evolution studies.

An alternative way to probe the end of the cosmic 'dark ages' and discriminate between different reionization histories is through $21 \mathrm{~cm}$ tomography. It has also long been known (e.g. Field 1959; Sunyaev \& Zel'dovich 1975; Hogan \& Rees 1979; Scott \& Rees 1990; Subramanian \& Padmanabhan 1993) that neutral hydrogen in the intergalactic medium (IGM) and gravitationally collapsed systems may be directly detectable in emission or absorption against the cosmic microwave background radiation $(\mathrm{CMB})$ at the frequency corresponding to the redshifted $21 \mathrm{~cm}$ line (associated with the spin-flip transition from the triplet to the singlet state). Madau, Meiksin, \& Rees (1997, hereafter MMR) first showed that $21 \mathrm{~cm}$ tomography could provide a direct probe of the era of cosmological reionization and reheating. In general, $21 \mathrm{~cm}$ spectral features will display angular structure as well as structure in redshift space due to inhomogeneities in the gas density field, hydrogen ionized fraction, and spin temperature. Several different signatures have been investigated in the recent literature: (a) the fluctuations in the $21 \mathrm{~cm}$ line emission induced by the 'cosmic web' that develops at early times in cold dark matter (CDM)-dominated cosmologies (Tozzi et al. 2000) and by 'minihalos' with virial temperatures below $10^{4} \mathrm{~K}$ (Iliev et al. 2002,2003); (b) the global feature ('reionization step') in the continuum spectrum of the radio sky that may mark the abrupt overlapping phase of individual intergalactic H II regions (Shaver et al. 1999); (c) and the $21 \mathrm{~cm}$ narrow lines generated in absorption against very high redshift radio sources by the neutral IGM (Carilli, Gnedin, \& Owen 2002) and by intervening minihalos and protogalactic disks (Furlanetto \& Loeb 2002).

In this paper, we use numerical simulations of hydrogen reionization by stellar sources in the context of $\Lambda \mathrm{CDM}$ cosmogonies to estimate the radio signal expected from the diffuse, low density IGM during the 'gray age' (Carilli et al. 2002). This is the era between the formation of the first luminous objects and the epoch of complete reionization, when the IGM is a mixture of neutral, partially ionized, and fully ionized structures. The important point is that the early generation of stars that ultimately ionizes the universe will also generate a background radiation field of $\operatorname{Ly} \alpha$ photons arising from their collective redshifted UV continua. It is the presence of a sufficient flux of Ly $\alpha$ photons that renders the neutral IGM 'visible' against

\footnotetext{
${ }^{1}$ Max-Planck-Institut für Astrophysik, Karl-Schwarzschild-Straße 1, 85748 Garching, Germany.

${ }^{2}$ Department of Astronomy \& Astrophysics, University of California, Santa Cruz, CA 95064.

${ }^{3}$ Osservatorio Astrofisico di Arcetri, Largo E. Fermi 5, 50125 Firenze, Italy.
} 
the CMB by mixing the hyperfine levels ('Ly $\alpha$ pumping'). Radio maps will show a patchwork (both in angle and in frequency) of emission signals from $\mathrm{H}$ I zones modulated by $\mathrm{H}$ II regions where no signal is detectable against the CMB.

The remainder of this paper is organized as follows. In $\S 2$ we summarize the physics of $21 \mathrm{~cm}$ excitation. In $\S 3$ we show that, since a 'typical' early stellar population will radiate a large number of UV continuum photons with energies between 10.2 and $13.6 \mathrm{eV}$ (hereafter $\operatorname{Ly} \alpha-\mathrm{c}$ ) to which the IGM is transparent, there will be an extended period of time prior to full reionization where fluctuations in the gas density and hydrogen ionized fraction may be detected at $21(1+z) \mathrm{cm}$. Numerical simulations of hydrogen reionization by Population III stars are presented in $\S 4$. Simulated radio maps are generated and the detectability of fluctuations in the sky brightness discussed in $\S 5$. Finally, in $\S 6$ we give our conclusions.

\section{LYMAN-ALPHA PUMPING}

A quick summary of the physics of $21 \mathrm{~cm}$ radiation will illustrate the basic ideas behind this work (see MMR for more details). The emission or absorption of $21 \mathrm{~cm}$ photons from a neutral IGM is governed by the hydrogen spin temperature, $T_{S}$, defined by

$$
n_{1} / n_{0}=3 \exp \left(-T_{*} / T_{S}\right),
$$

where $n_{0}$ and $n_{1}$ are the number densities of atoms in the singlet and triplet $n=1$ hyperfine levels and $T_{*}=$ $0.068 \mathrm{~K}$ is the excitation temperature of the transition. In the presence of only the CMB radiation with $T_{\mathrm{CMB}}=$ $2.73(1+z) \mathrm{K}$, the spin states will reach thermal equilibrium with the $\mathrm{CMB}$ on a timescale of $T_{*} /\left(T_{\mathrm{CMB}} A_{10}\right) \approx$ $3 \times 10^{5}(1+z)^{-1}$ yr $\left(A_{10}=2.85 \times 10^{-15} \mathrm{~s}^{-1}\right.$ is the spontaneous decay rate of the hyperfine transition of atomic hydrogen), and intergalactic $\mathrm{H}$ I will produce neither an absorption nor an emission signature. Thus, a mechanism is required that decouples $T_{S}$ and $T_{\mathrm{CMB}}$, e.g. by coupling the spin temperature instead to the kinetic temperature $T_{K}$ of the gas itself. Two mechanisms are available, spinexchange collisions between hydrogen atoms (Purcell \& Field 1956) and scattering by Ly $\alpha$ photons (Wouthuysen 1952; Field 1958; Field 1959). The first process proceeds at a rate that is too small for realistic IGM densities at the redshifts of interest, although collisions will be important in dense regions with gas overdensities $\delta \gtrsim 20[(1+z) / 10]^{-2}$, like virialized minihalos (Iliev et al. 2002,2003; Furlanetto \& Loeb 2002). It is Ly $\alpha$ pumping that dominates instead in the diffuse IGM, by mixing the hyperfine levels of neutral hydrogen in its ground state via intermediate transitions to the $2 p$ state, the WouthuysenField process. In this case,

$$
P_{\alpha}=\frac{4 \pi J_{\alpha} \sigma_{\alpha}}{h_{P} \nu_{\alpha}},
$$

is the rate at which Ly $\alpha$ photons of energy $h_{P} \nu_{\alpha}$ in an isotropic background of intensity $J_{\alpha}$ are scattered by an $\mathrm{H}$ atom in the gas, and

$$
P_{\mathrm{th}} \equiv \frac{27 A_{10} T_{\mathrm{CMB}}}{4 T_{*}}=7.6 \times 10^{-13}(1+z) \mathrm{s}^{-1},
$$

is the critical ('thermalization') scattering rate which, if much exceeded, would drive $T_{S}$ away from $T_{\mathrm{CMB}}$ and towards $T_{K}$ (MMR). In the above equations $\sigma_{\alpha}=\int \sigma_{\nu} d \nu=$ $\pi e^{2} f_{\alpha} / m_{e} c=0.011 \mathrm{~cm}^{2} \mathrm{~Hz}$ is the integrated Ly $\alpha$ scattering cross section. The condition $P_{\alpha}>P_{\text {th }}$ can also be rewritten as

$$
J_{\alpha}>J_{\mathrm{th}}=9 \times 10^{-23}(1+z) \operatorname{ergscm}{ }^{-2} \mathrm{~s}^{-1} \mathrm{~Hz}^{-1} \mathrm{sr}^{-1} .
$$

At $z=8$ this thermalization rate corresponds to $P_{\mathrm{th}} \nu_{\alpha} /\left(c n_{\mathrm{H}} \sigma_{\alpha}\right) \lesssim 1 / 2$ Ly $\alpha$ photons per hydrogen atom, where $n_{\mathrm{H}}$ is the intergalactic mean hydrogen density.

\section{UV SPECTRA OF EARLY STELLAR POPULATIONS}

At the epochs of interest, Ly $\alpha$ pumping will efficiently decouple $T_{S}$ from $T_{\mathrm{CMB}}$ if $J_{\alpha} \gtrsim 10^{-21} \mathrm{ergs} \mathrm{cm}^{-2} \mathrm{~s}^{-1} \mathrm{~Hz}^{-1}$ $\mathrm{sr}^{-1}$. The issue is then whether this critical value of $J_{\alpha}$ will be reached well before the epoch of complete reionization, when large regions of the IGM are still neutral. The process of reionization begins as individual sources drive isolated $\mathrm{H}$ II regions in the surrounding IGM and ends when these ionized bubbles overlap and fill the intergalactic volume. The volume filling factor of $\mathrm{H}$ in regions in a clumpy medium reaches unity when about $1+t / \bar{t}_{\text {rec }}$ ionizing photons have been emitted per hydrogen atom, where $\bar{t}_{\mathrm{rec}}$ is the volume-averaged hydrogen recombination timescale (Madau, Haardt, \& Rees 1999). Based on the density field in large scale cosmological simulations, only a few Lyman-continuum (Lyc) photons per hydrogen atom may be needed (Gnedin 2000; Miralda-Escudé, Haehnelt, \& Rees 2000) to keep the gas in mildly overdense regions and intergalactic filaments ionized against radiative recombinations at $z \sim 7$; many photons, however, will not escape local absorption, i.e. only a small fraction, $f_{\text {esc }}$, of the Lyc radiation emitted by massive stars is expected to escape the dense sites of star formation into galaxy halos and the intergalactic space. Moreover, at the earliest epochs of structure formation in CDM cosmologies the smallest nonlinear objects are the numerous halos that condense with masses just above the cosmological Jeans mass. Such 'minihalos' are not yet fully resolved nor is the process of their photoevaporation captured in large-scale three-dimensional cosmological simulations. This sub-grid structure makes the treatment of IGM clumping in all presently available simulations only approximate (Haiman, Abel, \& Madau 2001). The error in small-scale clumping (that leads to underestimating the total recombination rate) can be partly compensated by the assumption of a small escape fraction.

The early generation of massive stars likely responsible for reionization will also generate a background radiation field of photons with energies between 10.2-13.6 $\mathrm{eV}$ to which the IGM is transparent. As each of these Ly $\alpha-c$ photons gets redshifted, it will ultimately reach the Ly $\alpha$ transition energy of $10.2 \mathrm{eV}$, scatter resonantly off neutral hydrogen, and mix the hyperfine levels. The observability of the pre-reionization IGM depends therefore on the UV spectrum of the firsts stars, i.e. on the number of Ly $\alpha-c$ photons emitted per H-ionizing photon, and on the escape fraction of Lyc photons into the IGM. Figure 1 shows the number of continuum photons per octave near the Ly $\alpha$ frequency emitted by a stellar population, 
$N_{\alpha}$. The UV spectral energy distribution is characterized by a strong Lyc break the size of which depends on age, initial mass function (IMF), metallicity, and star formation history. We have therefore normalized $N_{\alpha}$ to the total number of hydrogen-ionizing photons emitted, $N_{\text {ion }}$. The values shown have been computed assuming a simple stellar population of metal-free 'Population III' stars: the simulations described in the next section use synthetic, time-dependent spectra of zero-metallicity stars to model the emission properties of stellar sources. For comparison, the figure also depicts the number of Ly $\alpha$ photons emitted by low metallicity stars $\left(Z=Z_{\odot} / 50\right)$. Note how the hotter temperatures of Population III stars produce a smaller Lyc break hence a smaller $N_{\alpha} / N_{\text {ion }}$ ratio. Also, after integrating over stellar age, a starburst is characterized by a ratio $N_{\alpha} / N_{\text {ion }}$ that is a few times higher than in the constant star formation rate case (this is because of the shorter lifetime of the massive stars that produce radiation above $1 \mathrm{ryd}$ ). It is possible that the starburst mode may be more relevant for the galaxies responsible for reionization, as stellar winds and supernovae can easily expel the gas out of the shallow potential wells of low mass halos, after the first burst of star formation occurs (Madau, Ferrara, \& Rees 2001).

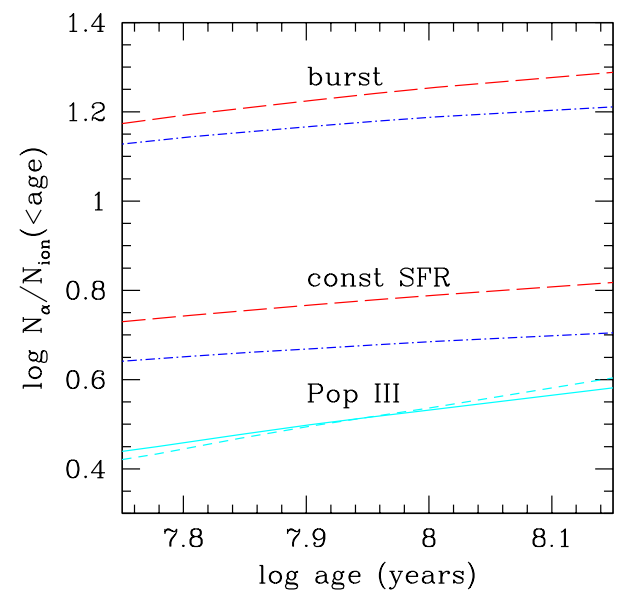

FIG. 1. - Number of continuum photons per octave in frequency just shortward of Ly $\alpha, N_{\alpha}$, emitted as a function of age by a stellar population with a Salpeter IMF (dot-dashed lines) and a Scalo (long-dashed lines) IMF. Upper set of curves assumes an instantaneous burst of star formation, lower set a constant star formation rate. We have normalized $N_{\alpha}$ to the number of hydrogen-ionizing photons per octave emitted just shortward of the Lyman limit, $N_{\text {ion }}$. The population synthesis values have been computed for low metallicities $\left(Z=0.02 Z_{\odot}\right)$, and are based on an update of Bruzual \& Charlot's (1993) libraries. Solid line: same for zero-metallicity Population III stars with a Salpeter IMF. Short-dashed line: same for zero-metallicity Population III stars with a Larson IMF (see Ciardi et al. 2001 and references therein).

As mentioned above, only an amount $N_{\text {phot }}=f_{\text {esc }} N_{\text {ion }}$ of Lyc photons will actually be able to escape from the galaxies and break into the IGM. A smaller escape fraction will generate a stronger Ly $\alpha$ diffuse radiation background for a given ionizing metagalactic flux. Taking a fiducial ratio $N_{\alpha} / N_{\text {ion }} \gtrsim 4$ and a few $\mathrm{H}$-ionizing photons per atom to keep the IGM fully photoionized, $N_{\text {phot }} / N_{\mathrm{H}} \approx 5$ (see Fig. 7 in Ciardi, Stoehr, \& White 2003, hereafter CSW), these simple estimates imply

$$
\frac{N_{\alpha}}{N_{\mathrm{H}}} \gtrsim 4 \frac{N_{\text {phot }}}{N_{\mathrm{H}}} f_{\mathrm{esc}}^{-1} \gtrsim 200\left(f_{\mathrm{esc}} / 0.1\right)^{-1}
$$

Ly $\alpha$ 'continuum' photons emitted per hydrogen atom close to the reionization epoch. This is more than two orders of magnitude larger than the thermalization rate for efficient level mixing by Ly $\alpha$ pumping. It appears then to be a generic prediction of cosmological reionization scenarios by stellar sources that the Lyo background from the early generation of stars which ultimately ionize the universe should be sufficiently intense to make intergalactic diffuse neutral hydrogen visible against the CMB well before the epoch of full reionization. As shown below, detailed simulations of IGM reionization that include cosmological radiative transfer effects will confirm this basic result.

\section{NUMERICAL SIMULATIONS OF IGM REIONIZATION}

In this and the following section we use the numerical simulations of hydrogen reionization by stellar sources described in CSW and Ciardi, Ferrara, \& White (2003, hereafter $\mathrm{CFW}$ ) to investigate the radio signal expected from the diffuse IGM prior to reionization. We give here a brief summary of the main features of the simulations, relevant to the present study. In the above papers, the reionization process is studied through a combination of high resolution N-body simulations (to describe the distribution of dark matter and diffuse gas, Springel, Yoshida, \& White 2001; Yoshida, Sheth, \& Diaferio 2001), a semi-analytic model of galaxy formation (to track gas cooling, star formation, and feedback from supernovae, Kauffmann et al. 1999; Springel et al. 2001) and the Monte Carlo radiative transfer code CRASH (to follow the propagation of ionizing photons into the IGM, Ciardi et al. 2001; Maselli, Ferrara, \& Ciardi 2003). The simulations, spanning the redshift range $z=6-20$, are performed for a $\Lambda$ CDM cosmology with $\left(\Omega_{m}, \Omega_{\Lambda}, \sigma_{8}, h, \Omega_{b}, n\right)=(0.3,0.7,0.9,0.7,0.04,1)$. The simulation box has a comoving length of $L=20 h^{-1} \mathrm{Mpc}$ and a particle mass of $M_{p}=1.7 \times 10^{8} h^{-1} \mathrm{M}_{\odot}$. This choice of the parameters allows to simulate a region of the universe with 'mean' properties, thus avoiding biases due to cosmic variance on small scales and, at the same time, to resolve the objects that produce the bulk of the ionizing radiation (Ciardi 2002). Each output of the simulation provides the gas distribution inside the box together with a galaxy catalog containing, among other quantities, their positions, stellar masses, and star formation rates. As discussed in $\S 5$, two sets of simulations will be used, with different choices for the galaxy emission properties. At each output, the propagation into the IGM of the ionizing photons emitted by the simulated galaxy population is followed with the radiative transfer code CRASH. The code has been tested to ensure a good convergence in the ionization fraction for a wide range of densities. The calculation is performed over a $N=128^{3}$ grid. The gas (pure hydrogen) distribution is assumed to track the dark matter. While radiation above $13.6 \mathrm{eV}$ from each source is fully absorbed at the ionization front surrounding it, the $\operatorname{Ly} \alpha-\mathrm{c}$ radiation is free to propagate and fill the space in between the $\mathrm{H}$ II regions. Since numerous sources are contributing to the $\operatorname{Ly} \alpha$ background throughout the universe, its flux is nearly isotropic in the cosmic frame of reference, and is 
given by

$$
J_{\alpha}(z)=\frac{c}{4 \pi} \int_{z}^{z_{\max }} \epsilon\left(\nu^{\prime}, z^{\prime}\right) \frac{(1+z)^{3}}{\left(1+z^{\prime}\right)^{3}}\left|\frac{d t}{d z^{\prime}}\right| d z^{\prime},
$$

where the proper emissivity $\epsilon\left(\nu^{\prime}, z^{\prime}\right)$ can be written as

$$
\epsilon\left(\nu^{\prime}, z^{\prime}\right)=\frac{\left(1+z^{\prime}\right)^{3}}{L^{3}} M_{*}\left(z^{\prime}\right) S\left(\nu^{\prime}\right) .
$$

Here, $\nu^{\prime} \equiv \nu_{\alpha}\left(1+z^{\prime}\right) /(1+z), M_{*}\left(z^{\prime}\right)$ is the total stellar mass in the simulation box at redshift $z^{\prime}$, and $S\left(\nu^{\prime}\right)$ is the time-dependent spectral energy distribution at frequency $\nu^{\prime}$. Since the UV photon production is rapidly decreasing with stellar age, $M_{*}\left(z^{\prime}\right)$ is the mass of newly (i.e. in the timescale between two outputs of the simulation) formed stars, i.e. we neglect the contribution from stars formed in previous outputs. In equation (6) we have assumed that Ly $\alpha$ photons are not attenuated by the intervening IGM, and that the IGM is opaque to ionizing photons prior to reionization, $z>z_{\text {ion }}$. Thus, the integration over redshift is carried out only up to $1+z_{\max }=4(1+z) / 3$. In Figure 2 the resulting Ly $\alpha$ background intensity is depicted as a function of redshift, together with the volumeaveraged ionization fraction $x_{v}$, for the two simulation runs. The figure clearly shows that the expected diffuse flux of Ly $\alpha$ photons should be sufficiently intense to decouple the spin temperature from the CMB during the 'grey age' from redshift $\sim 20$ to complete reionization.

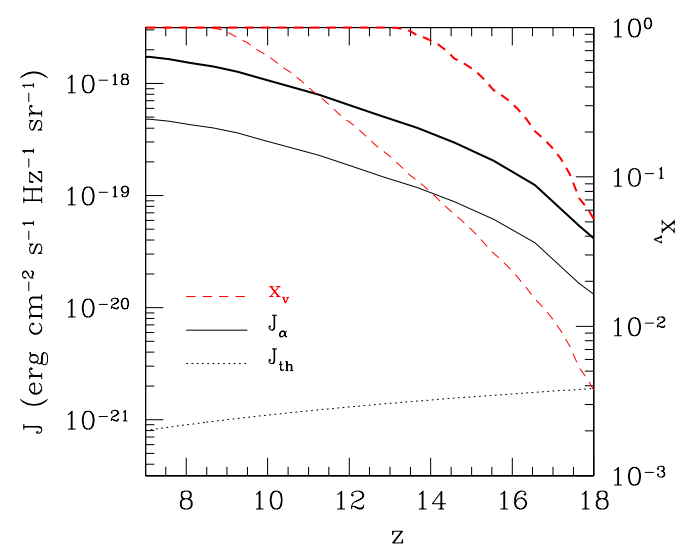

FIG. 2.- Solid lines: redshift evolution of the 'continuum' Ly $\alpha$ background, $J_{\alpha}$, predicted by our simulations of cosmological reionization by stellar sources. Dashed lines: volume-averaged ionization fraction, $x_{v}$. For each set of curves, the thick (thin) line refers to the 'early reionization' ('late reionization') scenario. Dotted line: Thermalization rate, $J_{\mathrm{th}}$, needed to efficiently decouple the spin temperature of neutral hydrogen from the CMB via Ly $\alpha$ pumping (see text for details).

\section{RADIO EMISSION FROM THE DIFFUSE IGM}

A beam of $21 \mathrm{~cm}$ radiation passing through a neutral hydrogen patch causes absorption and induces emission. In the radio range the exponential in equation (1) is close to unity and stimulated emission cancels most of the absorption. The $21 \mathrm{~cm}$ absorption coefficient depends then on the spin temperature. If the patch is located at redshift $z$, has (mean) spin temperature $T_{S}$, cosmic overdensity $\delta$, neutral hydrogen fraction $(1-x)$, angular diameter on the sky larger than the beamwidth of the radio telescope, and a radial velocity width broader than the bandwidth, its optical depth at $21(1+z) \mathrm{cm}$ is given by (MMR)

$$
\tau=\frac{3}{32 \pi} \lambda_{10}^{3} A_{10} \frac{T_{*}}{T_{S}}(1+\delta)(1-x) \frac{n_{\mathrm{H}}}{H} \simeq 0.006 \mathcal{T} \frac{T_{\mathrm{CMB}}}{T_{S}},
$$

where we have included in the function $\mathcal{T}$ all dependences on the density and ionization state of the patch, and on cosmological parameters,

$$
\mathcal{T} \equiv \frac{1}{h}(1+\delta)(1-x)\left(\frac{\Omega_{b} h^{2}}{0.02}\right)\left[\left(\frac{1+z}{10}\right)\left(\frac{0.3}{\Omega_{m}}\right)\right]^{1 / 2} .
$$

Here the Hubble constant is $H_{0}=100 h \mathrm{~km} \mathrm{~s}^{-1} \mathrm{Mpc}^{-1}$ and we have used the approximation $H(z) \approx \sqrt{\Omega_{m}} H_{0}(1+$ $z)^{3 / 2}$.

Because of the weakness of the magnetic hyperfine transition, $\tau$ will typically be much less than unity. In the rest frame of the patch, the radiative transfer equation yields for the brightness temperature through the translucent IGM $T_{b}=T_{\mathrm{CMB}} e^{-\tau}+T_{S}\left(1-e^{-\tau}\right)$. The flux observed at Earth can be expressed by the differential antenna temperature between this patch and the $\mathrm{CMB}$, $\delta T_{b}=\left(T_{b}-T_{\mathrm{CMB}}\right)(1+z)^{-1}$ or

$$
\delta T_{b} \simeq \frac{T_{S}-T_{\mathrm{CMB}}}{1+z} \tau \simeq 0.016 \mathrm{~K} \mathcal{T}\left(1-\frac{T_{\mathrm{CMB}}}{T_{S}}\right) .
$$

If $P_{\alpha}>P_{\mathrm{th}}$ and the IGM has been preheated by primordial sources of radiation, $T_{S}$ will be much larger than $T_{\mathrm{CMB}}$, and the universe will be observable in $21 \mathrm{~cm}$ emission at a level that is independent of the exact value of $T_{S}$. If preheating is negligible, the adiabatic expansion of the universe will lower the kinetic (hence spin) temperature of the gas well below that of the CMB, and the IGM will be detectable in absorption against the CMB. The energetic demand for heating the IGM above the CMB temperature is meager, only $\sim 0.004 \mathrm{eV}$ per particle at $z \sim 10$. Consequently, even relatively inefficient heating mechanisms may be important warming sources as structure develops before the universe was actually reionized, such as Ly $\alpha$ heating (MMR) and X-ray heating (MMR; Venkatesan, Giroux, \& Shull 2001) in low density regions, and shock heating of the gas in high density regions (Carilli et al. 2002). In the cosmological simulations of Carilli et al. (2002), the IGM is reionized 'late' but the volume-averaged kinetic and spin temperatures of the gas exceed significantly $T_{\mathrm{CMB}}$ already at redshifts 14 and 10 , respectively. In the following we will focus on the radio emission signal expected from our simulations in the limit $T_{S} \gg T_{\mathrm{CMB}}$.

\section{1. 'Late reionization' model}

The first simulation describes a 'late reionization' scenario in which the galaxy emission properties are computed assuming a Salpeter IMF, a time-dependent spectrum typical of metal-free stars, and an escape fraction $f_{\text {esc }}=5 \%(\mathrm{CSW}$; run S5 in CFW). The choice of these 


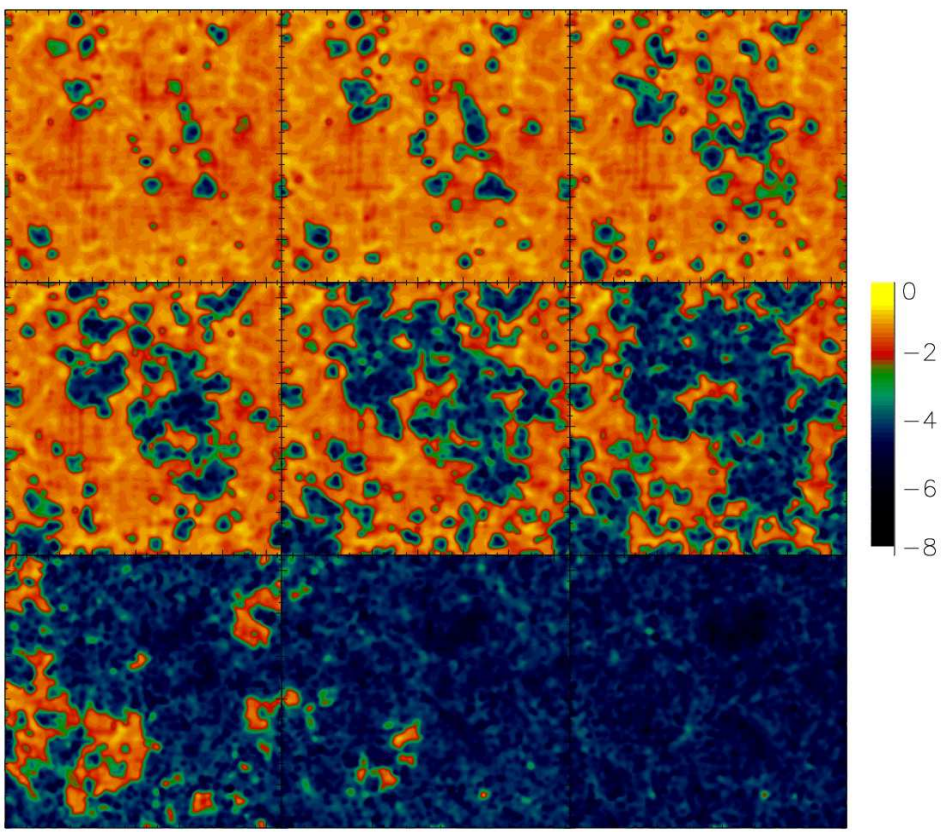

FIG. 3.- Slices through the simulation box in the 'late reionization' model. The nine panels show the differential antenna temperature $\log \left(\delta T_{b} / \mathrm{K}\right)$ at redshifts (from top to bottom and left to right) $z=13.5,12.8,12.0,11.3,10.6,9.9,9.3,8.7$, and 8.1. The maps refer to the emission produced by a slice of gas of comoving thickness $L / N^{1 / 3}=220 \mathrm{kpc}$.

parameters leads to a reionization epoch $z_{\text {ion }} \approx 8$ (defined here as the redshift where the volume-averaged ionization fraction, $x_{v}$, reaches unity). We use the expressions derived in equations (8)-(10) together with our simulations to account for the spatial distribution of structures and their different ionization levels during the reionization process. Maps of the antenna temperature can be thus constructed at each redshift based on equation (10), with $\mathcal{T}$ in equation (9) derived from the simulations along each line-of-sight. In Figure 3, the resulting $\delta T_{b}$ maps are shown for different redshifts. The maps refer to the emission produced by a slice of the simulation box of comoving thickness $L / N^{1 / 3}=220 \mathrm{kpc}$. Highly ionized regions (the dark areas in the map) initially occupy only a small fraction of the volume. At redshift 12, when about $90 \%$ of the IGM is still neutral, $\delta T_{b}$ reaches values $\sim 0.1 \mathrm{~K}$ in mildly overdense regions with $\delta$ greater than a few: the antenna temperature drops rapidly with cosmic time as reionization proceeds. By redshift of 11 or so, several bubbles have already overlapped as the first sources of ionizing photons are highly clustered. Finally, by $z=8.7$ most of the volume has been photoionized with only a few dense $\mathrm{H}$ I patches surviving.

The distribution of $\delta T_{b}$ values is shown in Figure 4. At $z=18.5$, most of the IGM is still neutral, and the brightness temperatures reflect density inhomogeneities still in the linear regime. As reionization gets under way, the distribution becomes double peaked due to the two-phase structure of the IGM: the rightmost peak corresponds to the neutral phase, while the left peak is associated with ionized gas. The pixel fraction corresponding to $\delta T_{b} \ll 10^{-8} \mathrm{~K}$ is not drawn in the figure: this increases by two orders of magnitude as $z_{\text {ion }}$ is approached. With decreasing redshift, more pixels are transferred from the right to the left peak. While the average $21 \mathrm{~cm}$ signal is two orders of magnitude lower than that of the CMB (and will be swamped by the much stronger non-thermal backgrounds that dominate the radio sky at meter wavelengths and that must be removed), its fluctuations - induced now both by inhomogeneities in the gas density and in the hydrogen ionized fraction - will greatly exceed those of the CMB. Brightness temperature fluctuations will be present both in frequency and in angle across the sky, and should be much easier to detect.

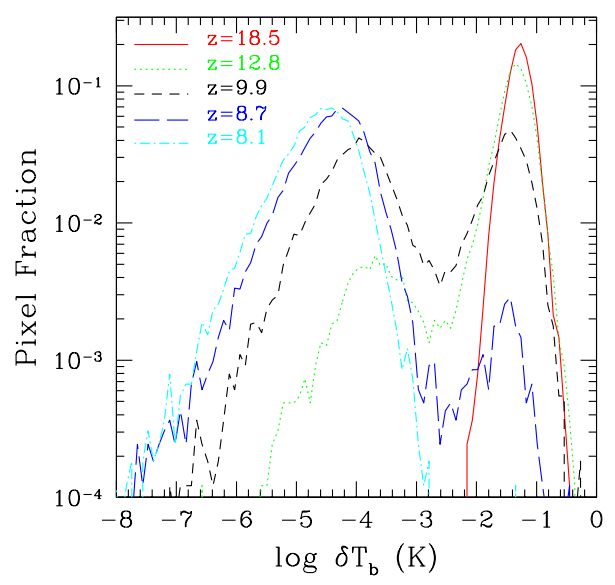

FIG. 4.- Distribution in our 'late reionization' simulation box of the differential antenna temperature $\delta T_{b}(\mathrm{~K})$ at redshifts $z=18.5$ (solid line), 12.8 (dotted line), 9.9 (small-dashed line), 8.7 (longdashed line) and 8.1 (dotted-dashed line). 


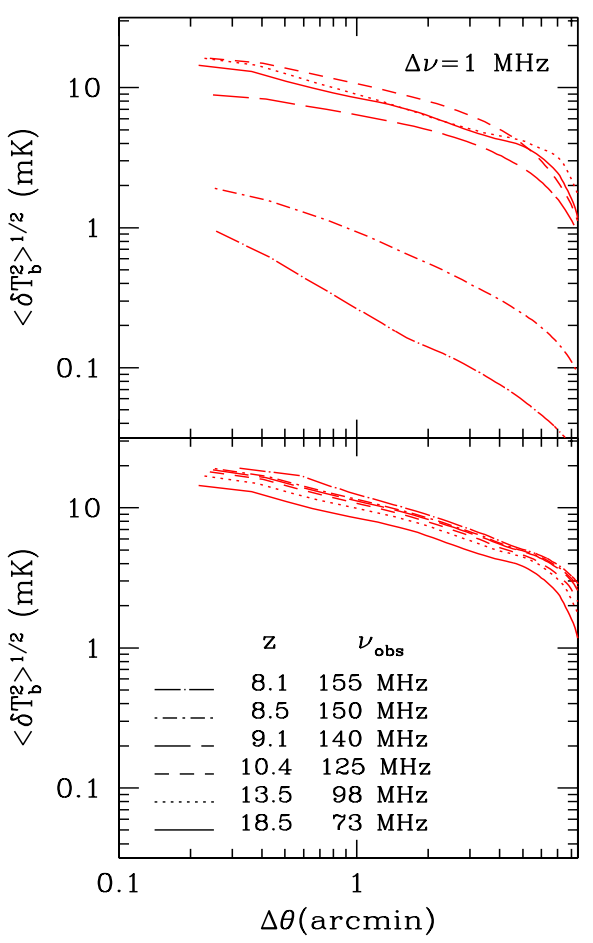

FIG. 5.- Upper panel: Expected rms brightness temperature fluctuations, $\left\langle\delta T_{b}^{2}\right\rangle^{1 / 2}$, in our 'late reionization' simulation box as a function of beam size $\Delta \theta$. A fixed bandwidth $\Delta \nu=1 \mathrm{MHz}$ has been assumed. Every curve corresponds to a different emission redshift or, equivalently, observed frequency $\nu_{\text {obs }}$. Lower panel: same in a test run with radiation sources switched off. Note that the box dimension is about 10 arcmin.

Figure 5 (upper panel) shows the expected rms temperature fluctuations relative to the mean, $\left\langle\delta T_{b}^{2}\right\rangle^{1 / 2}$, produced by a gas volume corresponding to a given bandwidth, $\Delta \nu=\nu_{\text {obs }} \Delta z /(1+z)$, and angular size $\Delta \theta$, as a function of $\Delta \theta$. The bandwidth is fixed for each redshift and equal to $\Delta \nu=1 \mathrm{MHz}$. The signal peaks at $z \sim 10.5(\sim 120 \mathrm{MHz})$, corresponding to the epoch when several high density neutral regions are still present, but $\mathrm{H}$ II occupies roughly half of the volume. As the mass variance is larger on small scales, at a fixed bandwidth $\left\langle\delta T_{b}^{2}\right\rangle^{1 / 2}$ increases with decreasing angular scale from about 5 to $20 \mathrm{mK}$.

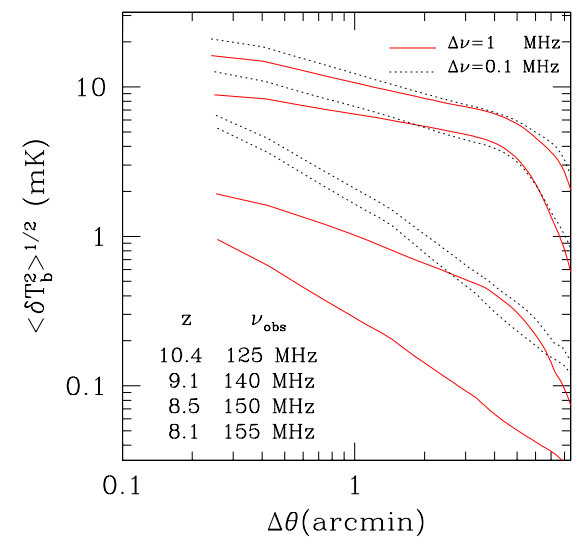

Fig. 6. - Same as the upper panel of Fig. 5 for two different bandwidths, $\Delta \nu=0.1$ (dotted lines) and $1 \mathrm{MHz}$ (solid).

At lower redshift $\left\langle\delta T_{b}^{2}\right\rangle^{1 / 2}$ decreases and it drops dramatically at $z \lesssim 8.5$, when the IGM gets close to complete reionization. Above redshift 11, the rms decreases again as the development of structures in the universe is less advanced. Although inhomogeneities in the ionized fraction drop to zero as the IGM is mostly neutral, a patchwork of radio emission is produced by regions with overdensities still in the linear regime and by voids. Note that the errors due to the box finiteness allow a correct evaluation of $\left\langle\delta T_{b}^{2}\right\rangle^{1 / 2}$ only for $\Delta \theta \lesssim 8 \mathrm{arcmin}$. To better gauge the suppression effect of cosmological reionization on the sky brightness at $21(1+z) \mathrm{cm}$, we have plotted in the lower panel the rms temperature fluctuations expected in the same simulation but with radiation sources switched off.



FIG. 7.- Expected rms brightness temperature fluctuations in the 'late reionization' case as a function of the observed frequency, $\nu_{o b s}$, for different choices of the beam angle, $\Delta \theta$. Notation is the same as in Fig. 5. The bandwidth is $\Delta \nu=1 \mathrm{MHz}$.

We have also checked that a smaller bandwidth of $\Delta \nu=0.1 \mathrm{MHz}$ gives roughly the same rms at high redshifts, while this becomes larger on small scales as $z$ decreases (Fig. 6). This is related to the fact that inhomogeneities in the gas density and in the hydrogen ionized fractions, which increase with decreasing redshift, are better resolved by a smaller bandwidth. The expected rms brightness temperature fluctuations as a function of the observed frequency, $\nu_{o b s}$, are shown in Figure 7 for our 'late reionization' run, for different choices of beam size, and for a bandwidth $\Delta \nu=1 \mathrm{MHz}$. The temperature fluctuations remain approximately constant over a wide range of redshift, drastically declining only when the IGM becomes highly ionized.

\section{2. 'Early reionization' model}

Recently, the discovery by the WMAP satellite of a large optical depth to Thomson scattering (Kogut et al. 2003; Spergel et al. 2003) suggests that the universe was reionized at $z_{\text {ion }}>10$. We have thus repeated the same analyses of $\S 5.1$ for a simulation in which the galaxy emission properties are computed assuming instead a Larson IMF (i.e. a 
Salpeter function with a cutoff below $\left.5 \mathrm{M}_{\odot}\right)$ and an escape fraction $f_{\text {esc }}=20 \%$ (run L20 in CFW). These parameters lead to an 'early reionization' epoch, $z_{\text {ion }} \approx 13$, more consistent with the WMAP observations (see CFW for an extensive discussion). The overall, qualitative results are the same described in the previous Section for the 'late reionization' case. Here, we limit our discussion to the expected value of the rms brightness temperature fluctuations, shown in Figure 8. The same curves in Figure 8 and Figure 5 (upper panel) correspond to redshifts of equivalent volume averaged ionization fraction, e.g., $x_{v} \sim 0.5$ is reached at $z=14.9$ (10.4) in the 'early (late) reionization' case and it corresponds to the short-dashed line in both Figures. The behavior is similar to the 'late reionization' model, except that now the neutral IGM is detectable only at radio frequencies below $100 \mathrm{MHz}$.

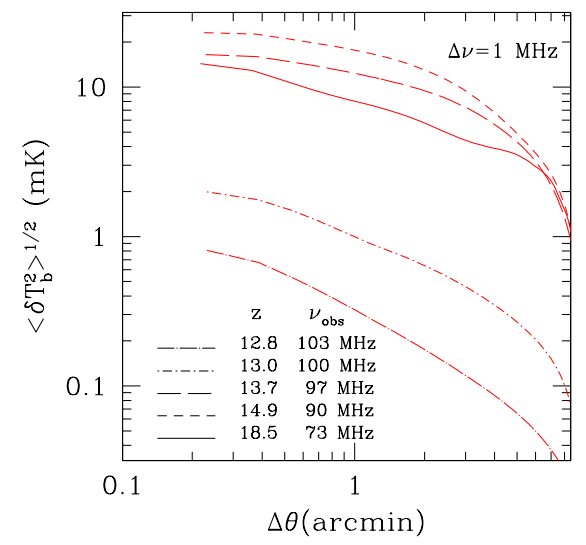

FIG. 8.- Same as in Fig. 5 (upper panel) but in the 'early reionization' case. Note how rms brightness temperature fluctuations on all scales now drop below $2 \mathrm{mK}$ at $z \lesssim 13$, making the IGM effectively undetectable in $21 \mathrm{~cm}$ radiation above radio frequencies of 100 MHz.

\section{DISCUSSION AND CONCLUSIONS}

In this paper we have used numerical simulations of hydrogen reionization by stellar sources in the context of a $\Lambda \mathrm{CDM}$ cosmogony to investigate the $21(1+z) \mathrm{cm}$ radio background expected prior to the epoch of cosmological reionization. Here, in addition to the emission induced by the 'cosmic web' (Tozzi et al. 2000), we can also estimate the contribution from inhomogeneities in the hydrogen ionized fraction. A further contribution comes from the minihalos with virial temperatures below the threshold for atomic cooling that form in abundance at high redshift, and are sufficiently hot and dense to emit collisionallyexcited $21 \mathrm{~cm}$ radiation (Iliev et al. 2002,2003). These halos are not resolved in our numerical simulations. While the combined signal from many minihalos within a sufficiently large volume is in principle detectable, the $21(1+\mathrm{z})$ $\mathrm{cm}$ flux from these objects will typically be dominated by the radio background from the neutral IGM (since the $\mathrm{H}$ I mass will always be larger in the non-collapsed phase; Oh \& Mack 2003), except in the very earliest stages of structure formation when $J_{\alpha}<J_{\mathrm{th}}$, i.e. at $z>20$. At $z>12$, the predicted brightness temperature fluctuations from minihalos on arcmin scales are typically much smaller than those expected from the diffuse IGM. As reionization proceeds, feedback effects (like photoevaporation) on minihalos may also decrease their expected $21 \mathrm{~cm}$ signal. Here, we have focused on emission from the diffuse, low density gas. The search at $21 \mathrm{~cm}$ for the epoch of first light has become one of the main science drivers of the LOw Frequency ARray $(L O F A R)$. Radio $21 \mathrm{~cm}$ tomography may probe the topology of reionization, map the cosmic 'gray age', i.e. the transition from the post-recombination universe to one populated with radiation sources, and effectively open up much of the universe to a direct study of the reheating and reionization epochs. On scales $\sim 5$ arcmin, predicted rms brightness temperature fluctuations can exceed $5 \mathrm{mK}$ for a $1 \mathrm{MHz}$ bandwidth. The expected sensitivity of LOFAR on these scales is about $10 \mathrm{mK}$ with a $1000 \mathrm{~h}$ integration (for a confidence level of 5 times the noise and $\Delta \nu=1$ $\mathrm{MHz}$, see http://www.astron.nl/lofar/science/), enough to detect all fluctuations above $2 \sigma$. While remaining an extremely challenging project due to foreground contamination from unresolved extragalactic radio sources (Di Matteo et al. 2002) and free-free emission from the same halos that reionize the universe (Oh \& Mack 2003), the detection and imaging of large-scale structure prior to reionization breakthrough remains a tantalizing possibility within range of the next generation of radio arrays.

The main results of this study can be summarized as follows.

- At epochs when the IGM is still mainly neutral, the simulated early galaxy population provides enough Ly $\alpha-$ c photons to decouple $T_{S}$ from $T_{\mathrm{CMB}}$. As in the same redshift range the IGM is expected to be 'warm', the $21 \mathrm{~cm}$ line would be seen in emission.

- The rms temperature fluctuations relative to the mean, $\left\langle\delta T_{b}^{2}\right\rangle^{1 / 2}$, increase with decreasing angular scale, as variance is larger on smaller scales. The signal peaks at an epoch when several high density neutral regions are still present, but $\mathrm{H}$ II occupies roughly half of the volume.

- Depending on the redshift of reionization breakthrough, broad-beam observations at frequencies $\lesssim$ $150 \mathrm{MHz}$ (below $100 \mathrm{MHz}$ for the 'early reionization' scenario) with the next generation of radio telescopes should reveal angular fluctuations in the sky brightness temperature in the range $5-20 \mathrm{mK}(1 \sigma)$ on scales $\lesssim 5$ arcmin.

We have benefited from discussions with T. Di Matteo, T. Ensslin, A. Ferrara, I. Iliev, M. Rees, and P. Tozzi. B.C. thanks F. Stoehr for helping with the simulations. Support for this work was provided by NSF grant AST0205738, by NASA grant NAG5-11513, and by a B. Rossi visiting fellowship at the Observatory of Arcetri (P.M.). B.C. acknowledges the support of the Research and Training Network "The Physics of the Intergalactic Medium" set up by the European Community under the contract HPRN-CT-2000-00126. 


\section{REFERENCES}

Becker, R. H., et al. 2001, AJ, 122, 2580

Bruzual, A. C., \& Charlot, S. 1993, ApJ, 405, 538

Carilli, C., Gnedin, N. Y., \& Owen, F. 2002, ApJ, 577, 22

Ciardi, B. 2002, in The Evolution of Galaxies. II. Basic Building Blocks, eds. M. Sauvage, G. Stazinska, \& D. Schaerer (Kluwer), p. 515

Ciardi, B., Ferrara, A., Marri, S., \& Raimondo, G. 2001, MNRAS, 324,381

Ciardi, B., Ferrara, A., \& White, S. D. M. 2003, MNRAS, submitted (astro-ph/0302451, CFW)

Ciardi, B., Stoehr, F., \& White, S. D. M. 2003, MNRAS, submitted (astro-ph/0301293, CSW)

Di Matteo, T., Perna, R., Abel, T., \& Rees, M. J. 2002, ApJ, 564, 576

Fan, X., et al. 2002, AJ, 123, 1247

Field, G. B. 1958, Proc. IRE, 46, 240

Field, G. B. 1959, ApJ, 129, 551

Furlanetto, S., \& Loeb, A. 2002, ApJ, 579, 1

Gnedin, N. Y. 2000, ApJ, 535, 530

Haiman, Z., Abel, T., \& Madau, P. 2001, 551, 599

Hogan, C. J., \& Rees, M. J. 1979, MNRAS, 188, 79

Iliev, I. T., Shapiro, P. R., Ferrara, A., \& Martel, H. 2002, ApJ, 572, L123

Iliev, I. T., Scannapieco, E., Martel, H., \& Shapiro, P. R. 2003, MNRAS, 341, 81
Kauffmann, G., Colberg, J. M., Diaferio, A., \& White, S. D. M. 1999, MNRAS, 303, 188

Kogut, A., et al. 2003, ApJ, submitted (astro-ph/0302213)

Madau, P., Ferrara, A., \& Rees, M. J. 2001, ApJ, 555, 92

Madau, P., Haardt, F., \& Rees, M. J. 1999, ApJ, 514, 648

Madau, P., Meiksin, A., \& Rees, M. J. 1997, ApJ, 475, 492 (MMR)

Maselli, A., Ferrara, A., \& Ciardi, B. 2003, MNRAS, submitted

Miralda-Escudé, J., Haehnelt, M., \& Rees, M. J. 2000, ApJ, 530, 1

Oh, S. P., \& Mack, K. J. 2003, MNRAS, submitted (astro$\mathrm{ph} / 0302099$

Purcell, E. M., \& Field, G. B. 1956, ApJ, 124, 542

Scott, D., \& Rees, M. J. 1990, MNRAS, 247, 510

Shaver, P., Windhorst, R., Madau, P., \& de Bruyn, G. 1999, A\&A, 345,380

Spergel, D.N., et al. 2003, ApJ, submitted (astro-ph/0302207)

Springel, V., White, S. D. M., Tormen, G., \& Kauffmann, G. 2001, MNRAS, 328, 726

Springel, V., Yoshida, N., \& White, S. D. M. 2001, NewA, 6, 79

Subramanian, K., \& Padmanabhan, T. 1993, MNRAS, 265, 101

Sunyaev, R. A., \& Zel'dovich, Y. B. 1975, MNRAS, 171, 375

Tozzi, P., Madau, P., Meiksin, A., \& Rees, M. J. 2000, ApJ, 528, 597

Venkatesan, A., Giroux, M. L., \& Shull, J. M. 2001, ApJ, 563, 1

Wouthuysen, S. A. 1952, AJ, 57, 31

Yoshida, N., Sheth, R. K., \& Diaferio, A. 2001, MNRAS, 328, 669 